\title{
Characteristics of Helicobacter pylori growth in a defined medium and determination of its amino acid requirements
}

\author{
David J. Reynolds and Charles W. Penn \\ Author for correspondence: David J. Reynolds. Tel: +4421 414 6563. Fax: +44214146557.
}

Microbial Molecular Genetics and Cell Biology Group, School of Biological Sciences, University of Birmingham, Birmingham B15 2TT, UK

\begin{abstract}
A defined medium has been developed for Helicobacter pylori that gives growth characteristics (growth rate, maximum cell number and maximum colony-forming-unit count) comparable to those in a complex medium (Isosensitest broth $+5 \%$, v/v, foetal bovine serum). Differences found in the death rate reflected a partial $(50 \%)$ conversion to a coccoid cell form of the organism in the stationary and death phase in the defined medium, versus the almost complete ( $>99 \%$ ) conversion seen in the complex medium. The medium was used to study the amino acids required for growth by 10 strains of $H$. pylori. All strains required arginine, histidine, isoleucine, leucine, methionine, phenylalanine and valine, and eight of the strains also required alanine; five of the strains required serine. In the absence of glucose none of the $\mathbf{2 0}$ amino acids tested elicited growth when added at high concentration. However, in the presence of glucose, alanine induced considerably enhanced growth over that seen in the control, consistent with its use either as a nitrogen source or possibly an additional carbon source. The medium described will facilitate investigations into the metabolism and physiology of $H$. pylori, previously only possible with sophisticated approaches such as nuclear magnetic resonance spectroscopy.
\end{abstract}

Keywords: Helicobacter pylori, metabolism, coccoid form, nutritional regulation

\section{INTRODUCTION}

Helicobacter pylori is now well established as a principal cause of gastritis (Marshall \& Warren, 1984; Morris \& Nicholson, 1987; reviewed by Robert \& Weinstein, 1993) and serological evidence shows $25-34 \%$ of the UK population and $52 \%$ of the population world-wide (ranging from $87 \%$ for Poland to $15 \%$ for Australia; Megraud, 1993) to be infected. Incidence of infection shows a high correlation with duodenal ulcer and to a lesser extent gastric ulcer and it is thought that chronic infection with $H$. pylori causes these lesions (Buck et al., 1986; Blaser, 1987, 1990; Hornick, 1987; Taylor \& Blaser, 1991). Furthermore, there is good epidemiological evidence to support the proposal that infection over a period of decades may be linked to the development of gastric cancer (Parsonnet et al., 1991; Nomura et al., 1991; Forman et al., 1991).

H. pylori has been widely studied since its discovery in 1982 (Warren \& Marshall, 1983) but many aspects of its structure, metabolism and physiology, including its specific growth requirements, are still largely unknown. The organism is generally grown in complex media supplemented with blood or serum, which has made the metabolic pathways utilized by the bacterium difficult to determine. For example, although early studies based on acid formation from sugars and detection of preformed enzymes found no evidence of saccharide fermentative pathways (Marshall \& Warren, 1984; McNulty \& Dent, 1987), more recent evidence indicates that $H$. pylori does catabolize sugars. In a series of studies by Mendz, Hazell and colleagues clear evidence has been obtained for the pentose phosphate pathway (Mendz \& Hazell, 1991), glucokinase activity (Mendz \& Hazell, 1993) and the fermentation of glucose to lactate (Mendz et al., 1993). Unfortunately the approach used by these workers, ${ }^{1} \mathrm{H}$ and ${ }^{13} \mathrm{C}$ nuclear magnetic resonance spectroscopy, is expensive to set up and use, which restricts its wider availability to researchers studying $H$. pylori metabolism.

Here we describe a simple medium in which all the constituents are defined, allowing each of them to be selectively omitted or further components added and the effect on growth studied. We have used this medium to determine the amino acid requirements of $H$. pylori and to 
Table 1. Composition of the defined medium

\begin{tabular}{|c|c|c|c|}
\hline Component & $\begin{array}{c}\text { Concn } \\
\left(\mathrm{mg} \mathrm{l}^{-1}\right)\end{array}$ & Component & $\begin{array}{c}\text { Concn } \\
\left(\mathrm{mg}^{-1}\right)\end{array}$ \\
\hline $\mathrm{Ca}\left(\mathrm{NO}_{3}\right)_{2} \cdot 4 \mathrm{H}_{2} \mathrm{O}$ & 100 & Amino acids & \\
\hline $\mathrm{KCl}$ & 400 & Alanine hydrochloride & $44 \cdot 5$ \\
\hline $\mathrm{MgSO}_{4} \cdot 7 \mathrm{H}_{2} \mathrm{O}$ & 100 & Arginine & 632 \\
\hline $\mathrm{NaCl}$ & 6000 & Asparagine & 75 \\
\hline $\mathrm{Na}_{2} \mathrm{HPO}_{4}$ & 800 & Aspartic acid & $66 \cdot 5$ \\
\hline $\mathrm{NaHCO}_{3}$ & 2000 & Cystine & 120 \\
\hline Glucose & 2000 & Glutamic acid & 73.5 \\
\hline \multirow[t]{2}{*}{ Bovine serum albumin } & 5000 & Glutamine & 300 \\
\hline & & Glycine & $37 \cdot 5$ \\
\hline Phenol red (optional) & 5 & Histidine & 110 \\
\hline \multirow[t]{2}{*}{$\mathrm{FeSO}_{4}$} & 2 & Isoleucine & $262 \cdot 5$ \\
\hline & & Leucine & 262 \\
\hline Adenine & 50 & Lysine & $362 \cdot 5$ \\
\hline \multirow[t]{2}{*}{ Lipoic acid } & 3 & Methionine & $75 \cdot 5$ \\
\hline & & Phenylalanine & 165 \\
\hline Vitamins & & Proline & $57 \cdot 5$ \\
\hline D-Biotin & $0 \cdot 2$ & Serine & $52 \cdot 5$ \\
\hline Choline chloride & 3 & Threonine & 238 \\
\hline Folic acid & 1 & Tryptophan & 51 \\
\hline myo-Inositol & 35 & Valine & 234 \\
\hline Niacinamide & 1 & & \\
\hline$p$-Aminobenzoic acid & 1 & & \\
\hline D-Pantothenic acid & $1 \cdot 25$ & & \\
\hline Pyridoxine hydrochloride & 1 & & \\
\hline Riboflavin & $0 \cdot 2$ & & \\
\hline Thiamin hydrochloride & 1 & & \\
\hline $\begin{array}{l}\text { Vitamin B12 } \\
\text { (cyanocobalamin) }\end{array}$ & $0 \cdot 005$ & & \\
\hline
\end{tabular}

study the utilization of amino acids as carbon and energy sources. In addition we have compared the growth pattern of the organism in the defined medium with that in a standard complex medium and looked in particular at the extent of coccoid cell formation in the two media.

\section{METHODS}

Strain and inoculum. The Helicobacter pylori strains used were NCTC 11637 (type strain), Roberts (clinical isolate from Manchester, UK) and eight isolates from stomach biopsies collected during 1991-1992 in Birmingham, UK. Other strains were Escherichia coli $\mathrm{K} 12$ and a Neisseria gonorrboeae strain with a requirement for the amino acids Cys, GIn and Arg (typed according to Catlin, 1973) provided by Dr N. Parsons, University of Birmingham, UK. All strains were maintained on Isosensitest agar (Unipath) containing 2\% (v/v) heat-inactivated newborn calf serum (Life Technologies). Plates were incubated at $37^{\circ} \mathrm{C}$ for a maximum of $3 \mathrm{~d}$ in a VAIN (variable atmosphere incubator set at $5 \% \mathrm{CO}_{2}, 5 \% \mathrm{O}_{2}$, balance $\mathrm{N}_{2}$; Don Whitley Scientific). Liquid cultures were grown in $15 \mathrm{ml}$ volumes of medium in $100 \mathrm{ml}$ conical flasks with gentle shaking (140 r.p.m., IKA Vibrax VXR; Janke \& Kunkel) or in $1 \mathrm{ml}$ volumes in the wells of a 24-well tray (NUNC) with vigorous shaking (240 r.p.m.). Inocula were grown overnight on agar plates then suspended in Hanks' balanced salts solution, washed once in the same solution and standardized to $1 \times 10^{8}$ cells $\mathrm{ml}^{-1}$.
Inoculation ( $150 \mu \mathrm{l}$ suspension for flasks, $10 \mu \mathrm{l}$ for trays) was carried out in a laminar-flow hood and the cultures immediately transferred to the VAIN incubator and shaken at $37^{\circ} \mathrm{C}$.

Chemicals. All the inorganic chemicals and glucose were obtained from BDH. The amino acids (tissue-culture grade), vitamins and other organic chemicals were from Sigma. The bovine serum albumin (BSA) was either from Sigma (Fraction V, catalogue no. A-7906) or from Advanced Protein Products, Brierley Hill, W. Midlands, UK (catalogue no. PF-201-47). Both were equally suitable, and were stored as a $14 \%(\mathrm{w} / \mathrm{v})$ aqueous solution at $4{ }^{\circ} \mathrm{C}$. Stock solutions were as follows (in water, sterilized by $0.2 \mu \mathrm{m}$ filtration and stored at $4{ }^{\circ} \mathrm{C}$, unless stated otherwise): amino acids, $100 \times$ concentration in medium (Table 1) in water or water acidified with $\mathrm{HCl}$, stored at $-20^{\circ} \mathrm{C}$; adenine, $6.5 \mathrm{mg} \mathrm{ml}^{-1}$ (heat needed to dissolve); $\mathrm{NaHCO}_{3}$, $7.5 \%$; inorganic salts were made as a single $4 \times$ solution; lipoic acid, $10 \mathrm{mg} \mathrm{ml}^{-1}$ in ethanol (not sterilized); glucose, $50 \times$ solution; vitamins, a single solution containing all 11 vitamins (Table 1) at $100 \times ; \mathrm{FeSO}_{4}, 100 \times$ in acidified water. The medium was made up to volume with de-ionized distilled water and the $\mathrm{pH}$ adjusted to $7 \cdot 4$.

Growth studies. Growth over time was assessed by measurement of $\mathrm{OD}_{600}$ using $1 \mathrm{ml}$ samples in semi-micro plastic cuvettes, by serial dilution viable count on Isosensitest agar plates and by phase-contrast microscopy using a Helber chamber (Weber Scientific). Proportions of coccoid and rod-shaped cells 
Relative growth

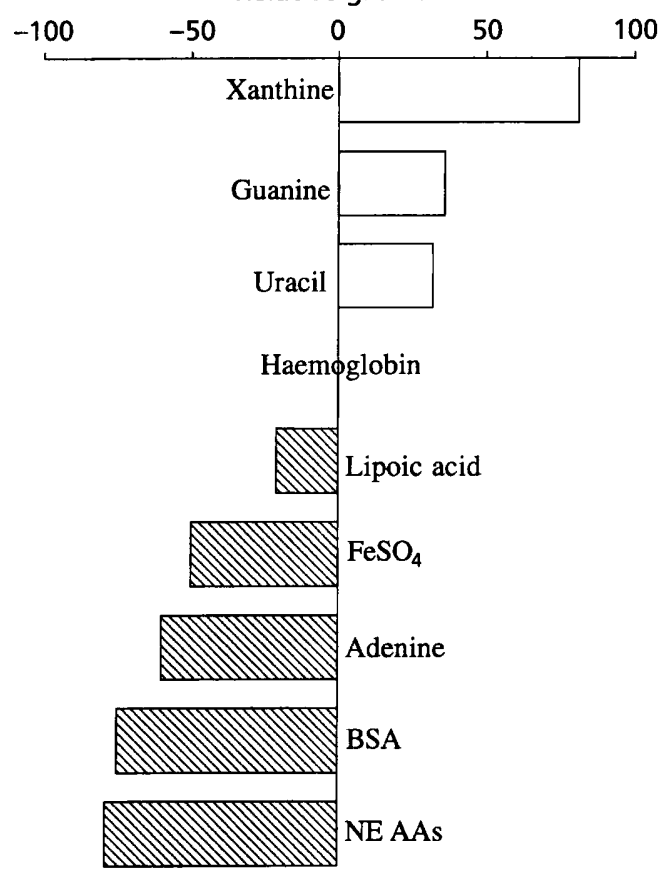

Fig. 1. Effect of component omission on growth of $H$. pylori. Each of the components shown in the figure was selectively omitted from the medium and the resulting growth at $41 \mathrm{~h}$ post-inoculation (measured as $O D_{600}$ ) recorded as a percentage change compared to the growth seen in the complete medium (no change $=0$ ). Where values are positive, growth is enhanced by component omission (a value of $+100 \%$ corresponds to an $O D_{600}$ of 0.423$)$; where negative, growth is suppressed by omission. The complete medium contained the components at the following concentrations: xanthine, guanine, uracil, adenine, $10 \mathrm{mg} \mathrm{l}^{-1}$; haemoglobin $14 \mathrm{mg} \mathrm{l}^{-1}$; $\mathrm{BSA} \mathrm{FeSO}_{4}$, lipoic acid and non-essential amino acids (NE AAs: Ala, Asp, Asn, Glu, Gly, Pro, Ser) at the concentrations shown in Table 1.

were assessed by phase-contrast examination of all 16 large squares (total area $1 / 400 \mathrm{~mm}^{2}$ ) of the Helber chamber (for two or three repeat assemblings of the chamber) at a dilution of the culture to give 10-20 organisms per square. All samples taken were confirmed as $H$. pylori by microscopic examination and comparison of their protein profile, using SDS-PAGE analysis on mini-gels, with that of the inoculum. Gels were run on BioRad mini-Protean II equipment using the conditions of Laemmli (1970) according to the manufacturer's instructions.

Amino acid studies. The amino acids required for growth were determined by inoculating wells of a 24-well tray in which each well contained medium deficient in one of the amino acids listed in Table 2. Growth was assessed at 24 and $48 \mathrm{~h}$; absence of growth after $48 \mathrm{~h}$ indicated that the missing amino acid was required for growth of that particular strain. To assess use of amino acids as a carbon and energy source, the concentrations of all 20 amino acids were reduced to a base level $(20 \%$ of the concentrations in the defined medium; Table 1) giving only limited growth (see Fig. 2) and individual amino acids added at 40 times the base level. Increased growth in the presence of the test amino acid indicated that it could be used as a carbon or energy source. Growth was determined visually, then as $\mathrm{OD}_{595}$, and confirmed as $H$. pylori by SDS-PAGE analysis. $\mathrm{OD}_{595}$ was measured in duplicate in Falcon 96-well flexible assay plates

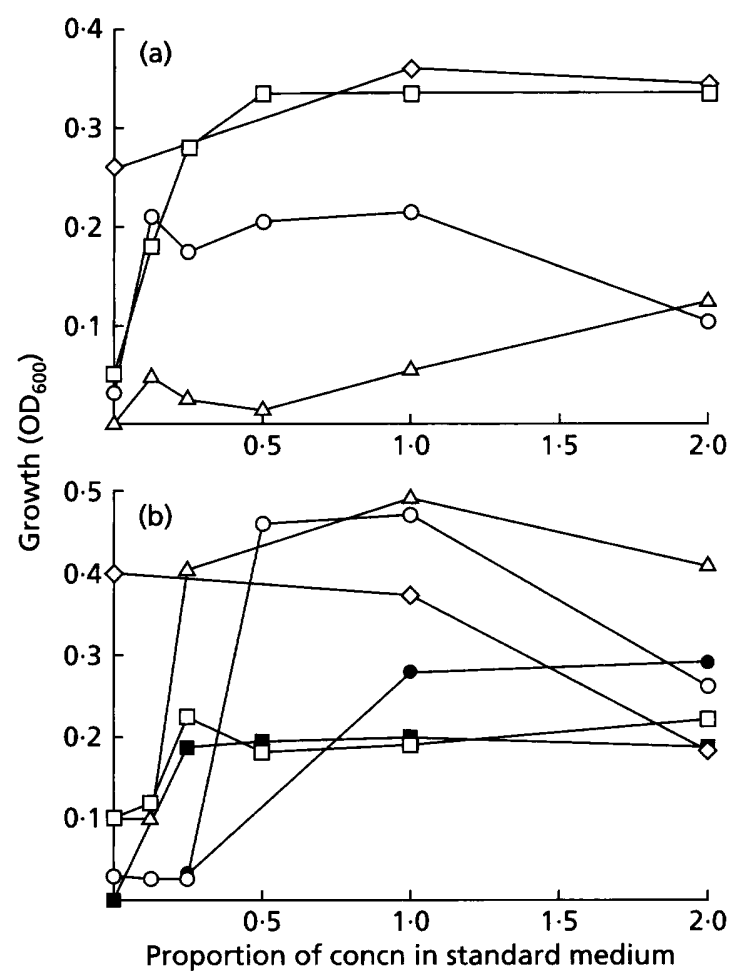

Fig. 2. Optimization of key media components. The concentrations of the components were individually altered from 0.125 times to twice the levels in the standard medium (Table 1) and the resulting growth measured as $\mathrm{OD}_{600}$ for strain NCTC 11637 (a) and strain Roberts (b). The components examined were: glucose $(\square)$, lipoic acid $(\diamond)$, adenine $(O)$, $\mathrm{FeSO}_{4}$ $(\triangle)$, BSA $(\square)$ and amino acids ( $\bullet$ ).

$(100 \mu \mathrm{l}$ per well) using a Titertek Multiskan MCC ELISA plate reader.

\section{RESULTS}

\section{Development of the defined medium}

Using tissue culture medium (RPMI, Life Technologies) as a basic chemically defined medium, a series of components was added until the medium would support growth. These components were chosen based on the published analysis of the Isosensitest medium used in this laboratory to grow $H$. pylori. The resulting supplemented medium was next prepared de novo from reagent-grade chemicals (BDH) and then simplified by sequentially removing each of the key ingredients and noting the effect on growth (Fig. 1). Reduction in growth in the absence of an ingredient indicated that it contributed towards growth in the complete medium whereas enhanced growth indicated that it had a deleterious effect. Xanthine, guanine, uracil and haemoglobin either failed to enhance or were detrimental to growth. Lipoic acid, $\mathrm{FeSO}_{4}$, adenine, BSA and non-essential amino acids increased growth of the organism. The concentrations of these key ingredients were then adjusted to determine the optimal medium composition (Fig. 2), resulting in the defined composite medium listed in Table 1. H. pylori 


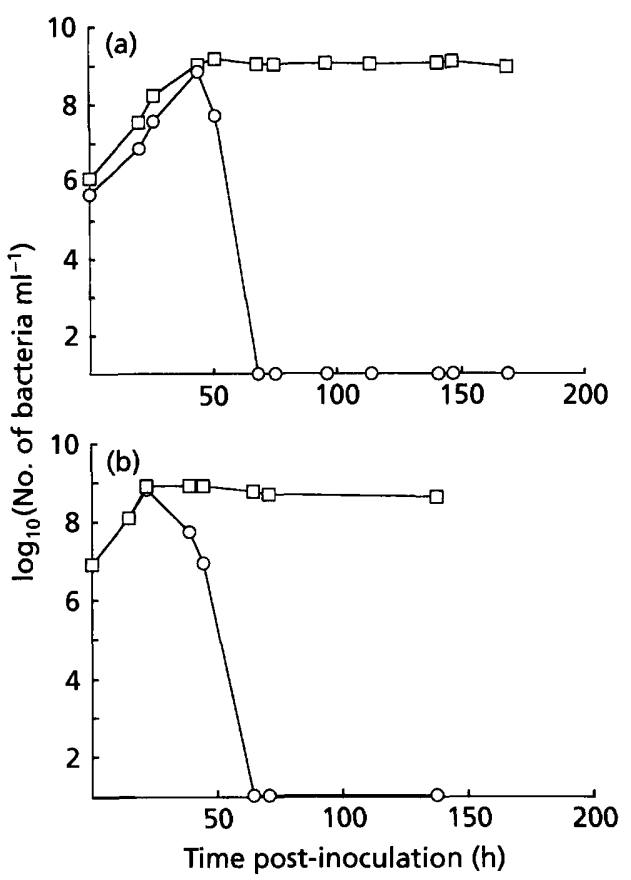

Fig. 3. Comparison of the growth of $H$. pylori in a complex medium and the defined medium. Growth was assessed as colony-forming units $(O)$ and total count $(\square)$ for strain Roberts grown in (a). Isosensitest broth $+5 \%$ foetal bovine serum and (b) the defined medium. The results are representative of three experiments.

strains Roberts and NCTC 11637 were both sequentially passaged in the medium five times and attained similar growth at each passage. A large inoculum was necessary to achieve growth during serial passage. Typically this was $0.75 \mathrm{ml}$ of a $24 \mathrm{~h}$ culture (approx. $2.5 \times 10^{7}$ bacteria) into $15 \mathrm{ml}$ medium and growth gave a 20 -fold increase in numbers of bacteria at each passage of $24 \mathrm{~h}$.

\section{Growth of $\boldsymbol{H}$. pylori in the defined medium}

Data are only shown for strain Roberts but results for NCTC 11637 were similar. The maximum growth rate was comparable for the defined and the complex media (Fig. 3; doubling time during exponential growth phase for defined medium $=2 \mathrm{~h} 33 \mathrm{~min}$, for complex medium $=2 \mathrm{~h} 37 \mathrm{~min}$ ). The maximum total counts and c.f.u. counts were also similar, at approximately $1 \times 10^{9}$ bacteria $\mathrm{ml}^{-1}$. There was a difference in the death rate: the mean halving time (time for the number of c.f.u. to halve) for the complex medium was lower than that for the defined medium ( $1 \mathrm{~h} 51 \mathrm{~min}$ compared to $2 \mathrm{~h} 4 \mathrm{~min}$, respectively), i.e. in the defined medium the bacterial became noncultivable less rapidly. The slower death of the culture in the defined medium was reflected in the rate of conversion to the non-cultivable coccoid form of the organism (Fig. 4). In the defined medium conversion was slower and only about $50 \%$ of the culture became coccoid even after prolonged incubation. In contrast in the complex medium

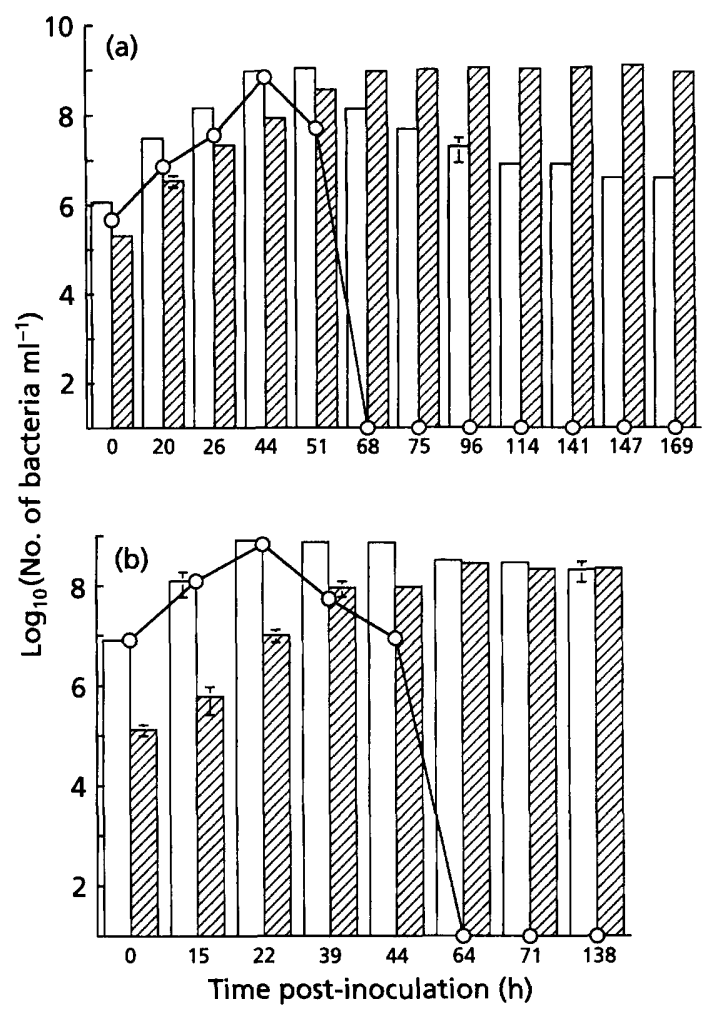

Fig. 4. Coccoid development in a complex medium (a) and the defined medium (b). Growth was assessed as colony-forming units $(O)$ and differential microscopic counts of the rod (clear columns) and coccoid (hatched columns) forms of the organism. The results are representative of two experiments. Bars represent SD.

conversion was rapid and over $99 \%$ of the culture became coccoid.

\section{Amino acids required for growth}

The amino acid requirements of 10 strains of $H$. pylori are shown in Table 2. All these strains required Arg, His, Ile, Leu, Met, Phe and Val. In addition, most strains also needed Ala and approximately half needed Ser, with only one requiring Pro and one Trp.

As a check on the validity of the procedure and media, two entirely distinct bacterial genera, represented by Neisseria gonorrboeae (known to require Cys, Gln and Arg) and Escherichia coli (a prototroph) were tested. Inocula were prepared as for $H$. pylori strains and the same media used. The amino acid requirements found matched the known requirements of the two strains, indicating the general applicability of the method.

\section{Amino acids as carbon and energy sources}

In the defined medium the amino acids are at relatively low levels and glucose is available as a carbon and energy source. To test whether individual amino acids could serve as alternatives to glucose, a glucose-free medium was made containing $20 \%$ of the normal medium 
Table 2. Amino acid requirements of $H$. pylori

+ , Growth in the absence of amino acid, i.e. amino acid not required; - , no growth in the absence of amino acid, i.e. amino acid required. The results are representative of four experiments for strains Roberts, NCTC 11637, HP79 and A654/91; two experiments for strains A681/91, Wedderburn, N. gonorrboeae and E. coli; and single experiments for the remaining strains.

\begin{tabular}{|c|c|c|c|c|c|c|c|c|c|c|c|c|c|c|c|c|c|c|c|c|}
\hline \multirow[t]{2}{*}{ Strain } & \multicolumn{12}{|c|}{ Amino acid: } & \multicolumn{8}{|c|}{ Amino acid: } \\
\hline & Asp & Asn & Cys & Gly & Glu & Gln & Lys & Pro & Ser & $\operatorname{Trp}$ & Thr & Tyr & Ala & Arg & His & Ile & Leu & Met & Phe & Val \\
\hline Roberts & + & + & + & + & + & + & + & + & + & - & + & + & - & - & - & - & - & - & - & - \\
\hline NCTC 11637 & + & + & + & + & + & + & + & + & + & + & + & + & - & - & - & - & - & - & - & - \\
\hline Troth & + & + & + & + & + & + & + & + & + & + & + & + & - & - & - & - & - & - & - & - \\
\hline A654/91 & + & + & + & + & + & + & + & + & + & + & + & + & - & - & - & - & - & - & - & - \\
\hline A655/92 & + & + & + & + & + & + & + & - & - & + & + & + & - & - & - & - & - & - & - & - \\
\hline A681/91 & + & + & + & + & + & + & + & + & + & + & + & + & - & - & - & - & - & - & - & - \\
\hline $\mathrm{HP} 2$ & + & + & + & + & + & + & + & + & - & + & + & + & + & - & - & - & - & - & - & - \\
\hline $\mathrm{HP} 79$ & + & + & + & + & + & + & + & + & + & + & + & + & + & - & - & - & - & - & - & - \\
\hline HP167 & + & + & + & + & + & + & + & + & - & + & + & + & - & - & - & - & - & - & - & - \\
\hline Wedderburn & + & + & + & + & + & + & + & + & - & + & + & + & - & - & - & - & - & - & - & - \\
\hline N. gonorrboeae & + & + & - & + & + & - & + & + & + & + & + & + & + & - & + & + & + & + & + & + \\
\hline E. coli & + & + & + & + & + & + & + & + & + & + & + & + & + & + & + & + & + & + & + & + \\
\hline
\end{tabular}

concentrations of amino acids and individual amino acids were then added at 20,40 or 100 times this level. No growth above that in the control (in which no amino acid was at high concentration) was seen for any of the amino acids for the four strains tested (HP79, A681/91, Roberts and NCTC 11637; data not shown). The experiments were then repeated using identical media supplemented with glucose (at $2 \mathrm{~g} \mathrm{l}^{-1}$ ) and a distinct pattern of growth was seen. For strains A681/91 and NCTC 11637 growth in the presence of the high level of amino acid was similar to that in the control but for strains Roberts and HP79 alanine induced a considerable increase in growth over that in the control (Fig. 5).

\section{DISCUSSION}

All of the media described to date for the cultivation of $H$. pylori have been complex media based on tissue extracts (e.g. of brain or heart) or proteolytic enzyme digests of meat or casein. Here we describe a defined medium capable of supporting growth comparable to that achieved in complex media.

Initial studies during the development of the defined medium highlighted lipoic acid, $\mathrm{FeSO}_{4}$, adenine, $\mathrm{BSA}$ and non-essential amino acids as key ingredients. Some of these are common additions to media used to grow other bacteria. However, the requirement for BSA, a protein known to act as a carrier for long-chain fatty acids (LCFAs), may indicate either that LCFAs are required by H. pylori if they are present in the BSA used in this study or that LCFAs are toxic and it is their removal by BSA that enhances growth. Alternatively, other required growth factors may be either carried by BSA or present as an impurity.

The pattern of growth in the defined and complex media was similar, with exponential growth to about $1 \times$
$10^{9}$ c.f.u. $\mathrm{ml}^{-1}$ followed by a short stationary phase and a rapid death phase. This is typical for other media studied, e.g. Mueller-Hinton broth (Sorberg et al., 1993) and Brucella broth (Catrenich \& Makin, 1991; Cellini et al., 1993). However, a major difference was that in the defined medium the rate of death in the death phase was lower and the conversion to the coccoid form reduced from $99 \%$ to just $50 \%$. This is a potentially important observation as it shows that conversion to the coccoid form can be regulated by changes induced by altered nutrition. Environmental factors are known to modulate the expression of virulence determinants (Mekalanos, 1992) and similar processes might also be involved in regulating the coccoid transformation. It is possible either that a key triggering component is at reduced abundance in the defined medium or that an $\mathrm{H}$. pylori metabolite necessary for the conversion is lowered by the lack of or reduced abundance of a nutritional factor.

Furthermore since the prevention of coccoid transition does not appear to increase the viability of the population in the death phase (c.f.u. count in both media $<10 \mathrm{ml}^{-1}$ ), it seems that the coccoid form is not a prerequisite for non-viability: rod forms of the organisms can also become non-viable.

The coccoid form of the organism has been proposed as a potential survival form possibly involved in transmission between individuals or reactivation of infection following apparently successful antibiotic therapy (Bode et al., 1993). The ability to manipulate transition to this form would be advantageous to workers studying this aspect of the organism.

The defined medium allowed the amino acid requirements to be determined by single amino acid omission. The requirement of eight amino acids for growth of $H$. pylori was the same as for the intracellular bacterium Legionella 


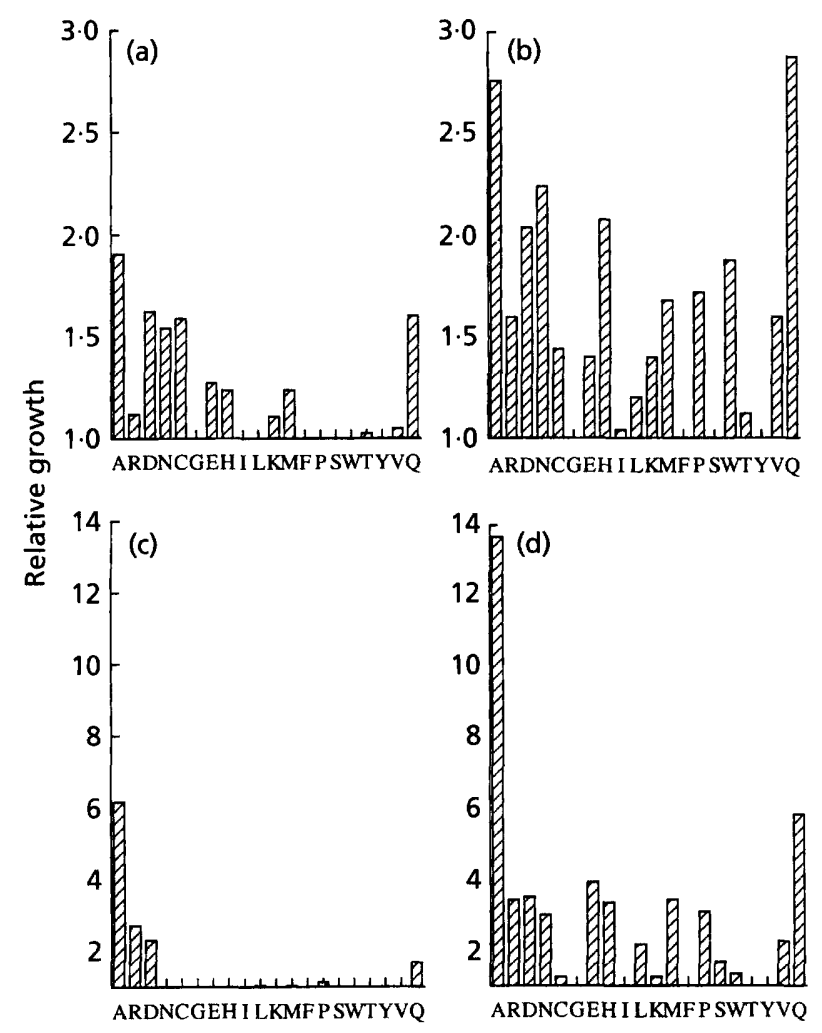

Fig. 5. Amino acids as carbon and energy sources for $H$. pylori. Individual amino acids (listed in the figure by their single-letter code) were added to the defined medium at $40 \times$ (results similar for $20 \times$ and $100 \times$ ) the levels in the low amino acid medium (glucose-free, containing $20 \%$ of the normal medium concentrations of amino acids) and the resulting growth (measured as $O D_{595}$ ) expressed as a multiple of the growth in the control (no amino acid at high level), where growth in the control $=1$. The strains tested were: (a) clinical isolate A681/91; (b) the type strain NCTC 11637; (c) clinical isolate Roberts; (d) clinical isolate HP79. The results are representative of two experiments (A681/91 and Roberts) or three experiments (NCTC 11637 and HP79).

pneumophila (George et al., 1980). Indeed six of the required amino acids (Arg, Ile, Leu, Val, Met and Phe) are the same for both bacteria. This contrasts with the 16 amino acids required by the lactic acid bacterium Leuconostoc mesenteroides (Lehninger, 1975) and the one to five amino acids commonly required by the pathogen Neisseria gonorrhoeae (usually selected from Cys, Pro, Arg, Gln, Ile and Met; Catlin, 1973). The amino acid requirements of $H$. pylori may form the basis of a scheme for biotyping strains of the bacteria given that there were differences in the requirements of the 10 strains tested here.

The amino acids that were required by $H$. pylori are significant in that they generally occur at the end of biosynthetic pathways, consistent with mutations in or deletions of the genes for individual enzymes in the pathways leading to their biosynthesis (Fig. 6). The $H$. pylori genome is only $1.72 \mathrm{Mbp}$ (cf. E. coli, which has a genome of $4.6 \mathrm{Mbp}$ ), suggesting that deletion of some non-essential genes has occurred. Mutations or deletions
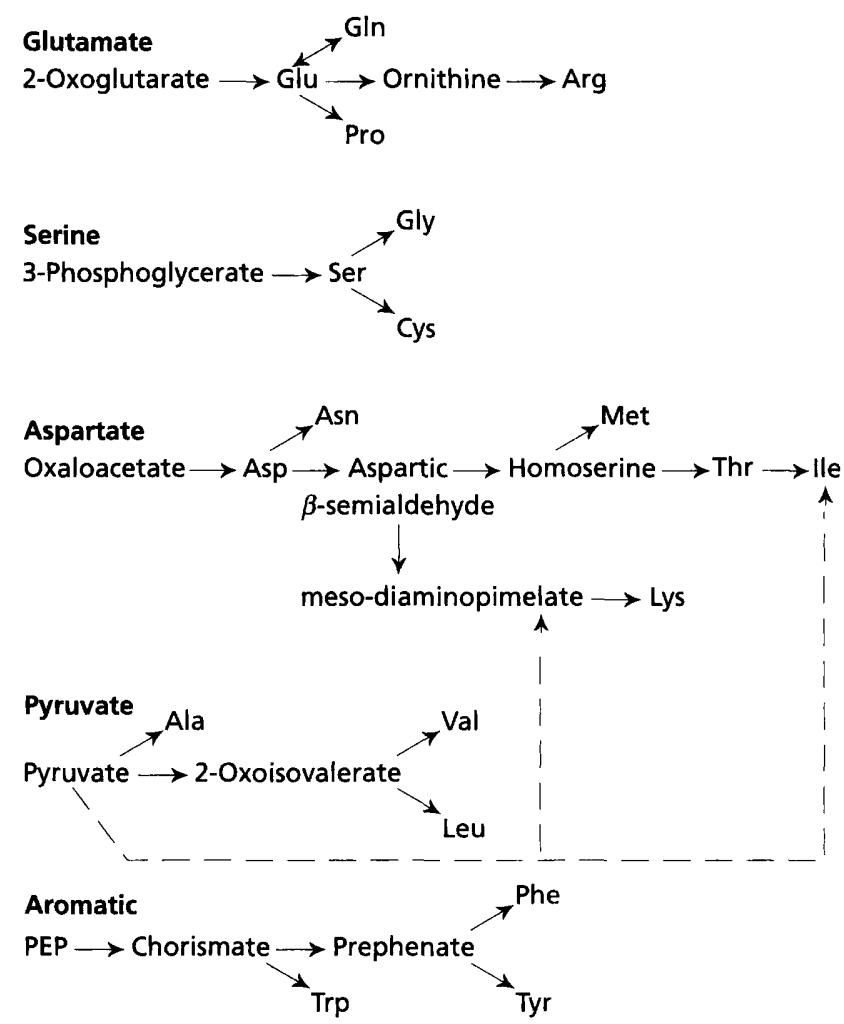

\section{Histidine}

Ribose-5-P + ATP $\longrightarrow$ His

Fig. 6. Biosynthetic pathway families for the synthesis of amino acids. Taken from Lehninger (1975).

at a point close to the root of a pathway would lead to multiple loss of biosynthetic capability and would probably have been too deleterious to permit survival of the organism in its natural habitat. One possible exception to this is the pyruvate pathway, where the ability to synthesize four amino acids has apparently been lost. However, this may simply reflect a weakness in the single amino acid omission analysis used as Leu, Ile and Val are structurally related amino acids known to be antagonistic in $E$. coli due to common active transport, biosynthesis and physiological regulation mechanisms and an imbalance in the supply of these amino acids can suppress growth (De Felice et al., 1979). The finding that four of the ten $H$. pylori strains required Ser is unusual in that this amino acid occurs at a fork in the biosynthetic pathway to Gly and Cys (Fig. 6). There is no requirement for either Gly or Cys in the presence of Ser so the enzymes responsible for biosynthesis downstream of Ser clearly exist. However, in the absence of Ser, growth is arrested despite the presence of Gly and Cys, indicating that there is a defect in the biosynthetic pathway to Ser. Loss of the ability to synthesize this amino acid would only permit survival if the organism had an adequate supply of Ser in its normal environment. The fact that this occurs for less 
than half of the strains tested suggests that $H$. pylori may be undergoing an evolutionary process of loss of the ability to synthesize amino acids.

Until recently it was generally thought that $H$. pylori did not use carbohydrates but was able to use amino acids as precursors for tricarboxylic acid cycle intermediates to derive energy (Dick, 1990). This is known to be the case for Legionella pneumophila, which uses Ser and Thr as carbon and energy sources (George et al., 1980). However, there is now strong evidence that $H$. pylori can utilize glucose as a carbon and energy source (see Introduction) and the data presented here suggest that amino acids alone cannot serve as carbon and energy sources for $H$. pylori.

In the absence of glucose the addition of single amino acids at high concentration did not enhance growth. However, in the presence of glucose several amino acids induced growth above that in the control for all four strains tested. For two of the strains (Roberts and HP79) the induction of growth in the presence of Ala was considerably greater than that seen for any of the other amino acids. This correlates with earlier evidence using proton nuclear magnetic resonance spectroscopy of brain heart infusion broth (a complex medium containing both amino acids and carbohydrates) which showed that Ala was metabolized by $H$. pylori (Dick, 1990). The requirement for the presence of glucose to permit growth enhancement by Ala may indicate that the amino acid serves as a carbon source but that its assimilation is driven by energy derived from glucose metabolism. Alternatively, the primary function of Ala catabolism may be to provide nitrogen with the carbon assimilation into tricarboxylic acid cycle derivatives noted by Dick (1990) being a consequence of amino acid deamination.

The experiments described here demonstrate the potential the defined medium has for elucidating the metabolic requirements of $H$. pylori and we intend in future work to use the medium to investigate the nutritional regulation of the virulence determinants of $H$. pylori. The medium may also be generally applicable to the growth of other helicobacters and the similar campylobacters; it clearly supports the growth of N. gonorrboeae. Modification of the medium to one in which all of the components are chemically defined is being pursued in this laboratory to allow us to dispense with the requirement for BSA.

\section{ACKNOWLEDGEMENTS}

We thank Richard Broadhead for his enthusiastic help during a post-examination project in this laboratory. This work was carried out under the tenure of post-doctoral fellowship (D. J. R.) from the AFRC (grant number LR6/552).

\section{REFERENCES}

Blaser, M. J. (1987). Gastric Campylobacter-like organisms, gastritis, and peptic ulcer disease. Gastroenterology 93, 371-383.

Blaser, M. J. (1990). Helicobacter pylori and the pathogenesis of gastroduodenal inflammation. J Infect Dis 161, 626-633.

Bode, G., Mauch, F. \& Malfertheiner, P. (1993). The coccoid forms of Helicobacter pylori. Criteria for their viability. Epidemiol Infect 111, $483-490$.
Buck, G. E., Gourley, W. K., Lee, W. K., Subramanyam, K., Latimer, J. M. \& Dinuzzo, A. R. (1986). Relation of Campylobacter pyloridis to gastritis and peptic ulcer. J Infect Dis 153, 664-669.

Catlin, B. W. (1973). Nutritional profiles of Neisseria gonorrboeae, Neisseria meningitidis and Neisseria lactamica in chemically defined media and the use of growth requirements for gonococcal typing. $J$ Infect Dis 128, 178-194.

Catrenich, C. E. \& Makin, K. M. (1991). Characterization of the morphologic conversion of Helicobacter pylori from bacillary to coccoid form. Scand J Gastroenterol (Suppl 181) 26, 58-64.

Cellini, L., Allocati, N., Di Campli, E., Masulli, M. \& Dainelli, B. (1993). Morphological forms in Helicobacter pylori. Acta GastroEnterol Belg Suppl 56, 108.

Defelice, M., Levinthal, M., laccarino, M. \& Guardiola, J. (1979). Growth inhibition as a consequence of antagonism between related amino acids: effect of valine in Escherichia coli K12. Microbiol Rev 43, $42-58$.

Dick, J. D. (1990). Helicobacter (Campylobacter) pylori: a new twist to an old disease. Annu Rev Microbiol 44, 249-269.

Forman, D., Newell, D. G., Fullerton, F., Yarnell, J. W. G., Stacey, A. R., Wald, N. \& Sitas, F. (1991). Association between infection with Helicobacter pylori and risk of gastric cancer: evidence from a prospective investigation. B Med J 302, 1302-1305.

George, J. R., Pine, L., Reeves, M. W. \& Harrell, W. K. (1980). Amino acid requirements of Legionella pneumopbila. J Clin Microbiol 11, 286-291.

Hornick, R. B. (1987). Peptic ulcer disease: a bacterial infection? $N$ Engl J Med 316, 1598-1600.

Laemmli, U. K. (1970). Cleavage of structural proteins during the assembly of the head of bacteriophage T4. Nature 227, 680685.

Lehninger, A. L. (1975). Biochemistry. New York: Worth Publishers. Marshall, B. J. \& Warren, J. R. (1984). Unidentified curved bacilli in the stomach of patients with gastritis and peptic ulceration. Lancet 2, 1311-1315.

McNulty, C. A. \& Dent, J. C. (1987). Rapid identification of Campylobacter pylori ( $C$. pyloridis) by preformed enzymes. J Clin Microbiol 25, 1683-1686.

Megraud, F. (1993). Epidemiology of Helicobacter pylori infection. In Helicobacter pylori Infection, pp. 73-88. Edited by C. P. Dooley \& H. Cohen. Philadelphia: Gastroenterology Clinics of North America.

Mekalanos, J. J. (1992). Environmental signals controlling expression of virulence determinants in bacteria. I Bacteriol 174, $1-7$.

Mendz, G. L. \& Hazell, S. L. (1991). Evidence for a pentose phosphate pathway in Helicobacter pylori. FEMS Microbiol Lett 84, 331-336.

Mendz, G. L. \& Hazell, S. L. (1993). Glucose phosphorylation in Helicobacter pylori. Arch Biochem Biophys 300, 522-525.

Mendz, G. L., Hazell, S. L. \& Burns, B. P. (1993). Glucose utilization and lactate production by Helicobacter pylori. J Gen Microbiol 139, 3023-3028.

Morris, A. \& Nicholson, G. (1987). Ingestion of Campylobacter pyloridis causes gastritis and raised fasting gastric $\mathrm{pH} . \mathrm{Am} J$ Gastroenterol 82, 192-199.

Nomura, A., Stemmermann, G. N., Chyou, P. H., Kato, I., PerezPerez, G. I. \& Blaser, M. J. (1991). Helicobacter pylori infection and gastric carcinoma among Japanese Americans in Hawaii. $N$ Engl $J$ Med 325, 1132-1136. 
Parsonnet, J., Friedman, G. D., Vandersteen, D. P., Chang, Y., Vogelman, J. H., Orentreich, N. \& Sibley, R. K. (1991). Helicobacter pylori infection and the risk of gastric carcinoma. N Engl J Med 325, $1127-1131$.

Robert, M. E. \& Weinstein, W. M. (1993). Helicobacter pyloriassociated gastric pathology. In Helicobacter pylori Infection, pp. 59-72. Edited by C. P. Dooley \& H. Cohen. Philadelphia: Gastroenterology Clinics of North America.

Sorberg, M., Nilsson, M. \& Nilsson, L. E. (1993). Morphologic conversion of Helicabacter pylori from bacillary form to coccoid form evaluated by bioluminescence, microscopy and viable count. Acta Gastro-Enterol Belg Suppl 56, 52.

Taylor, D. N. \& Blaser, M. J. (1991). The epidemiology of Helicobacter pylori infection. Epidemiol Rev 13, 42-59.

Warren, J. R. \& Marshall, B. J. (1983). Unidentified curved bacilli on gastric epithelium in active chronic gastritis. Lancet ii, 1273 1275 .

Received 11 May 1994; revised 24 June 1994; accepted 30 June 1994. 